Gizeli de F́́tima Ribero dos Anjos'

Angélica Lemos Debs Diniz ${ }^{2}$

Maria Céla dos Santos ${ }^{2}$

Nicholas Godor Canazza Damian ${ }^{3}$

Artigo Original

Palavras-chave

Artéria oftálmica/ultrassonografia Diabetes gestacional/fisiopatologia

Gestação

Hemodinâmica

Diabetes mellitus

Velocidade do fluxo sanguíneo

Keywords

Ophthalmic artery/ultrasonography Diabetes, gestational/physiopathology

Pregnancy

Hemodynamics

Diabetes mellitus

Blood flow velocity

\title{
Estudo do perfil hemodinâmico da artéria oftálmica em gestantes com diabetes mellitus gestacional
}

\author{
Study of ophthalmic artery bemodynamic pattern in pregnant \\ women with gestational diabetes mellitus
}

\section{Resumo}

OBJETIVOS: Avaliar os padrões hemodinâmicos da artéria oftálmica, por meio da doplervelocimetria, em portadoras de diabetes mellitus gestacional (DMG), e comparar os achados com os obtidos em gestantes normais. MÉTODOS: Estudo prospectivo caso-controle que analisou os índices doplervelocimétricos das artérias oftálmicas. Foram constituídos dois grupos: um com 40 gestantes com diagnóstico de DMG e o outro por 40 gestantes normais. Foram incluídas gestantes com DMG segundo critérios da American Diabetes Association - 2012, com gravidez de 27 semanas até o termo, e excluídas gestantes hipertensas, em uso de drogas vasoativas ou com diagnóstico prévio de diabetes. $\bigcirc$ estudo foi realizado em um olho com transdutor linear de $10 \mathrm{MHz}$ Sonoace 8000 Live Medison ${ }^{\circledR}$. As variáveis analisadas foram: índice de pulsatilidade (IP), índice de resistência (IR), razão entre picos de velocidade (RPV), pico de velocidade sistólica (PVS) e velocidade diastólica final (VDF). Para análise de normalidade das amostras foi empregado o teste de Lillefors, e os testes $t$ de Student e Mann-Whitney, para comparação entre as médias e medianas conforme normalidade dos dados, e adotado nível de significância de 95\%. RESULTADOS: As medianas e médias com desvio padrão das variáveis doplervelocimétricas da artéria oftálmica dos grupos de DMG e gestantes normais

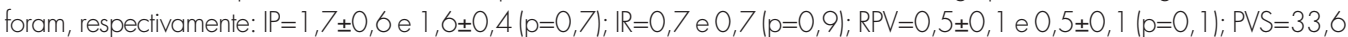
e 31,9 cm/seg $(p=0,7) ; V D F=6,3$ e 7,9 cm/seg $(p=0,4)$. Não houve diferença significante entre as médias e medianas das variáveis analisadas entre os dois grupos de gestantes. CONCLUSÕES: Os padrões hemodinâmicos das artérias oftálmicas permaneceram inalterados no grupo de gestantes com DMG em relação ao grupo de gestantes normais, sugerindo que o tempo de exposição à doença durante a gestação é curto para causar vasculopatias importantes no território materno central.

\section{Abstract}

PURPOSES: To evaluate the hemodynamic patterns of the ophthalmic artery by Doppler analysis in women with gestational diabetes mellitus (GDM), comparing them to normal pregnant women. METHODS: A prospective case-control study that analyzed the ophthalmic artery Doppler indices in two groups: one consisting of 40 women diagnosed with GDM and the other of 40 normal pregnant women. Included were pregnant women with GDM criteria of the American Diabetes Association - 2012, with 27 weeks of pregnancy to term, and excluded were women with hypertension, use of vasoactive drugs on or previous diagnosis of diabetes. Doppler analysis was performed in one eye with a $10 \mathrm{MHz}$ linear transducer and the Sonoace 8000 Live Medison ${ }^{\circledR}$ equipment. The following variables were analyzed: pulsatility index (PI), resistance index (RI), peak velocity ratio (PVR), peak systolic velocity (PSV) and end diastolic velocity (EDV). To analyze the normality of the samples we used the Lillefors test, and to compare means and medians we used the Student's ttest and Mann-Whitney test according to data normality, with the level of significance set at 95\%. RESULTS: The median and mean values with standard deviation of the variables of the ophthalmic artery Dopplervelocimetry group GDM and normal pregnant women were: $\mathbb{P}=1.7 \pm 0.6$ and $1.6 \pm 0.4(p=0.7) ; \mathbb{R}=0.7$ and $0.7(p=0.9) ; R P V=0.5 \pm 0.1$ and $0.5 \pm 0.1(p=0.1), P S V=33.6$ and $31.9 \mathrm{~cm} / \mathrm{sec}(p=0.7) ; V D F=6.3$ and $7.9 \mathrm{~cm} / \mathrm{sec}$ $(p=0.4)$. There was no significant difference in the means and medians of these variables between the two groups of pregnant women. CONCLUSIONS: The ophthalmic artery hemodynamic patterns, analyzed by means of a Doppler technique remained unchanged in the group of pregnant women with GDM compared to the group of normal pregnant women, suggesting that the time of exposure to the disease during pregnancy was too short to cause significant vascular disorders in the central territory.

Correspondência Angélica Lemos Debs Diniz Avenida Pará, 1720 - Bairro Umuarama CEP: $38400-902$ Uberlândia (MG), Brasil

Recebido

$17 / 01 / 2012$
Trabalho realizado no Departamento de Ginecologia e Obstetrícia da Universidade Federal de Uberlândia - UFU - Uberlândia (MG), Brasil.

Setor de Alto Risco, Universidade Federal de Uberlândia- UFU - Uberlândia (MG), Brasil.

2Departamento de Ginecologia e Obstetrícia da Faculdade de Medicina, Universidade Federal de Uberlândia - UFU - Uberlândia (MG), Brasil. ${ }^{3}$ Setor de Ultrassom, Universidade Federal de Uberlândia - UFU - Uberlândia (MG), Brasil.

Conflitos de interesse: não há 
Introdução

$\mathrm{O}$ diabetes mellitus compreende um grupo heterogêneo de distúrbios metabólicos de etiologias variáveis, caracterizado pela presença de hiperglicemia acompanhada de alterações no metabolismo dos carboidratos, gorduras e proteínas, decorrentes tanto do defeito na secreção quanto da ação da insulina. A classificação atual, segundo a American Diabetes Association, inclui quatro classes: diabetes tipo 1, diabetes tipo 2, diabetes secundário e diabetes gestacional. Esse último é definido como intolerância à glicose de graus variáveis, com início ou reconhecimento pela primeira vez durante a gestação, podendo ou não persistir após o parto ${ }^{1}$.

$\mathrm{O}$ diabetes mellitus gestacional (DMG) constitui atualmente um problema de saúde pública tanto em países desenvolvidos quanto em desenvolvimento. Nos Estados Unidos estima-se que $8 \%$ das 4 milhões de gestações estejam associadas ao diabetes. Dessas, $88 \%$ apresentam diabetes gestacional e $12 \%$, diabetes pré-gestacional ${ }^{2}$. $\mathrm{O}$ número de mulheres com diabetes na gravidez tem aumentado, em parte, como reflexo do aumento de mulheres obesas em idade fértil ${ }^{3}$.

Sabe-se que o diabetes mellitus leva a alterações vasculares importantes e, portanto, representa riscos à saúde da mãe e do feto. As complicações vasculares evidenciadas nessa doença geralmente são decorrentes das modificações endoteliais e da reatividade anormal das arteríolas ante o estado hiperglicêmico materno persistente ${ }^{4,5}$. Entretanto, as pacientes portadoras de DMG têm seu estado hiperglicêmico limitado à segunda metade da gestação e, portanto, postula-se que não haveria tempo de injúria suficiente para que as modificações da micro e macrovasculatura materna se instalassem.

Um dos métodos disponíveis atualmente para o estudo da hemodinâmica arterial do corpo humano é a doplervelocimetria. Tendo em vista que o diabetes mellitus é uma doença sistêmica que acomete vários órgãos e sistemas, torna-se importante o reconhecimento dos padrões hemodinâmicos das artérias, tanto do sistema nervoso central quanto do território periférico da gestante ${ }^{6}$.

A análise dos vasos orbitais vem ganhando destaque no contexto da Medicina moderna, tanto na Oftalmologia quanto na Obstetrícia. O aprimoramento da técnica Doppler permitiu o estudo do comportamento hemodinâmico de artérias do sistema nervoso central pela análise das artérias retrobulbares, em especial a artéria oftálmica. Essa artéria faz parte do sistema vascular central e é de topografia periférica; portanto, tem similaridades embriológicas, anatômicas e funcionais com os vasos de pequeno calibre do sistema nervoso central ${ }^{7}$. Visto que a artéria oftálmica dá origem aos vasos que formam a circulação orbital, ela é o principal vaso utilizado no estudo doplervelocimétrico do leito orbital ${ }^{8}$.
O interesse em se estudar a doplervelocimetria da artéria oftálmica em gestantes diabéticas deve-se à possibilidade de o método identificar alterações na microvascularização orbital obtidas por meio da análise quantitativa da onda de velocidade de fluxo (OVF) desta artéria. Assim, é possível mensurar a impedância e o grau de perfusão em um determinado território analisando-se os valores dos índices doplervelocimétricos disponíveis após registro da OVF.

Encontramos apenas um artigo disponível na literatura que avaliou o índice de pulsatilidade (IP) da artéria oftálmica em dois grupos de gestantes: um grupo de pacientes diabéticas tipo 1 e outro Grupo Controle, sendo ambos os grupos compostos por pacientes normotensas. Os autores descreveram sinais de hiperperfusão e vasodilatação orbital no grupo de diabéticas e redução do IP no grupo de diabéticas em relação aos índices do Grupo Controle, indicando compensação vascular antes do desenvolvimento da angiopatia em gestantes com diabetes mellitus do tipo $1^{8}$.

Em relação aos padrões hemodinâmicos das artérias centrais de gestantes portadoras de DMG, ainda não há estudos na literatura. Não se sabe se a exposição temporária à hiperglicemia é capaz de modificar a impedância nas artérias retrobulbares maternas.

O objetivo deste estudo foi analisar os padrões hemodinâmicos da artéria oftálmica, por meio da doplervelocimetria, em grávidas com diagnóstico de diabetes mellitus gestacional em relação a um Grupo Controle de gestantes normais.

\section{Métodos}

Trata-se de um estudo caso-controle prospectivo que analisou e comparou os índices doplervelocimétricos da artéria oftálmica entre dois grupos: um composto por 40 pacientes com diabetes mellitus gestacional e outro composto por 40 gestantes normais.

As pacientes foram selecionadas no ambulatório de pré-natal de alto e baixo risco do Hospital de Clínicas da Universidade Federal de Uberlândia, no período de abril de 2010 a abril de 2011 e são oriundas de uma mesma região metropolitana. Foram selecionadas 112 pacientes de forma consecutiva. Dentre as pacientes selecionadas, uma não compareceu ao seguimento pré-natal, 11 não retornaram no período puerperal, 11 desenvolveram pré-eclâmpsia ao longo do pré-natal, duas foram classificadas como portadoras de diabetes mellitus do tipo 2 no puerpério e 7 apresentaram intolerância à glicose no puerpério tardio, após a sexta semana do parto.

O projeto de pesquisa foi aprovado pelo Comitê de Ética em Pesquisa da Universidade Federal de Uberlândia, sob o Parecer $n^{\circ} 293 / 10$. As pacientes foram informadas sobre o caráter da pesquisa e manifestaram, por escrito, sua concordância em participar de estudo, pelo termo de 
consentimento livre e esclarecido. Os critérios de inclusão estabelecidos estão descritos a seguir.

Mulheres do Grupo Controle: gestantes que tiveram a avaliação clínico-laboratorial no pré-natal de baixo risco e preencheram todos os critérios de normalidade, tanto maternos, quanto fetais, idade gestacional acima de 27 semanas calculada com base na ultrassonografia do primeiro trimestre, feto vivo e único. Todas as gestantes foram acompanhadas até o puerpério tardio e não apresentaram nenhuma intercorrência nesse período.

Para o Grupo de Estudo (Grupo DGM) foram selecionadas gestantes com diagnóstico de DMG, gestação única, feto vivo e sem anomalias estruturais e com idade gestacional de mais que 27 semanas, calculada com base na ultrassonografia realizada no primeiro trimestre. Os critérios diagnósticos de DMG serão descritos a seguir. Foram excluídas do grupo de estudo gestantes portadoras de diabetes mellitus prévio, cirurgia ocular prévia, cardiopatias que pudessem alterar o ritmo cardíaco, tabagismo, uso de drogas ilícitas, hipertensão arterial sistêmica, pré-eclâmpsia, lúpus eritematoso sistêmico. Todas as grávidas foram submetidas ao rastreamento universal do diabetes pela realização do exame de glicemia de jejum na primeira consulta de pré-natal. O ponto de corte foi de $85 \mathrm{mg} / \mathrm{dL}$. Gestantes com valores acima deste foram submetidas à realização de teste oral de sobrecarga à glicose após a ingestão de $75 \mathrm{~g}$ de glicose. Adotamos os valores de corte estabelecidos pela $\mathrm{ADA}^{1}: 92 \mathrm{mg} / \mathrm{dL}, 180 \mathrm{mg} / \mathrm{dL}$ e $153 \mathrm{mg} / \mathrm{dL}$ para jejum, uma e duas horas, respectivamente. O diagnóstico do DMG foi firmado pelo encontro de um ou mais valores acima dos valores de corte durante a gestação ${ }^{1}$, seguido de confirmação com identificação do teste de tolerância a sobrecarga de glicose normal seis semanas após o parto.

O método laboratorial para avaliação de glicemia foi da Hexoquinase/Glicose-6-fosfato desidrogenase com o equipamento Architeet C8000 (ABBOT).

A média da idade das gestantes estudadas com DMG foi de 30,0 0 5,5 anos e das gestantes normais foi $24,2 \pm 5,5$ anos. A média das idades gestacionais foi $33,9 \pm 2,7$ semanas e 33,7 $\pm 2,7$ semanas nos grupos de grávidas normais e diabéticas, respectivamente. A média da pressão arterial no grupo de gestantes com DMG e Grupo Controle foi 10x6 mmHg. Os dois grupos foram pareados somente pela idade gestacional e pressão arterial. As idades maternas foram inferiores a 36 anos.

Para a realização do estudo doplervelocimétrico das artérias oftálmicas foi empregado o equipamento ultrassonográfico Sonoace 8000 da Medison ${ }^{\circledR}$, Estados Unidos, com transdutor linear na frequência de $10 \mathrm{MHz}$. Foram estudadas as seguintes variáveis da artéria oftálmica: índice de pulsatilidade (IP); índice de resistência (IR); pico de velocidade sistólica (PVS); pico de velocidade mesodiastólica razão entre picos de velocidade (RPV) e velocidade diastólica final (VDF).

A técnica utilizada para análise da artéria oftálmica foi a descrita em $2004^{7}$, pela qual o exame foi realizado com a paciente em decúbito dorsal, após 10 minutos de repouso. $\mathrm{O}$ transdutor era posicionado transversalmente sobre a pálpebra superior com os olhos fechados, após a colocação de uma pequena quantidade de gel. Foram realizados movimentos em sentido crânio-caudal, identificando-se o vaso, por meio de Doppler colorido, sem pressionar o transdutor sobre a pálpebra, a fim de evitar alterações dos dados doplervelocimétricos obtidos.

A artéria oftálmica era observada medialmente ao nervo óptico, e seu fluxo, registrado a $15 \mathrm{~mm}$ do disco óptico. Após a identificação da artéria, acionou-se o Doppler pulsado (espectral), sendo registradas seis ondas sem mudanças do padrão, para posterior aferição dos índices doplervelocimétricos em uma onda. $\mathrm{O}$ local de análise da artéria era sempre o mesmo para cada paciente da pesquisa.

A aferição dos índices de resistência e pulsatilidade, pico de velocidade sistólica, razão entre picos de velocidade e velocidade diastólica final foi efetuada em apenas um olho . Para evitar artefatos de espectros de velocidade, o ângulo de amostra de volume da doplervelocimetria foi sempre inferior a 20 graus. Em todos os casos, o filtro Doppler foi ajustado em $50 \mathrm{HZ}$, para evitar a perda do componente diastólico do fluxo. Foram determinadas a frequência de repetição de pulsos em $5,5 \mathrm{kHz}$ e a amostra volume em $2 \mathrm{~mm}$. Os cálculos dos índices doplervelocimétricos foram obtidos automaticamente pelo equipamento de ultrassonografia, exceto a RPV, proposto para análise de padrões dicróticos, calculada a partir da fórmula $\mathrm{RPV}=\mathrm{P} 2 / \mathrm{P} 1$, onde $\mathrm{P} 1$ representa o pico de velocidade sistólica e P2 é o pico de velocidade mesodiastólica após a incisura protodiastólica. O pico de velocidade mesodiastólica foi calculado pela medida da primeira elevação diastólica da onda de velocidade do fluxo,expressa em centímetros por segundo $(\mathrm{cm} / \mathrm{s})$.

Os dados foram apresentados de forma descritiva, utilizando a mediana, média e desvio padrão. $\mathrm{O}$ cálculo amostral foi feito baseado na prevalência do DMG de aproximadamente $7 \%$, utilizando nível de confiança de $95 \%$. Foram empregados para análise de normalidade das amostras o teste de Lillefors e os testes $t$ de Student e Mann-Whitney para comparação entre as médias e medianas, conforme normalidade dos dados. Empregou-se nível de confiança de $95 \%$ ( $\mathrm{p}<0,05)$, com intervalo de confiança de $95 \%$.

\section{Resultados}

Os dois grupos de gestantes estudadas não apresentaram diferenças significantes entre as médias das idades 
Tabela 1. Medianas, Médias e desvio padrão dos índices doplervelocimétricos da artéria oftálmica nos grupos de pacientes normais e diabéticas gestacionais

\begin{tabular}{lccccc}
\hline Grupos & $\mathbb{I R}^{* *}$ & $\mathbb{I} \mathbf{P}^{*}$ & $\begin{array}{c}\text { PVS** } \\
(\mathrm{cm} / \mathrm{s})\end{array}$ & RPV $^{*}$ & $\begin{array}{c}\text { VDF** } \\
(\mathrm{cm} / \mathrm{s})\end{array}$ \\
\hline Controle & 0,7 & $1,6 \pm 0,4$ & 31,9 & $0,5 \pm 0,1$ & 7,9 \\
DMG & 0,7 & $1,7 \pm 0,6$ & 33,6 & $0,5 \pm 0,1$ & 6,3 \\
Valor $\mathrm{p}$ & 0,9 & 0,7 & 0,7 & 0,1 & 0,4 \\
\hline
\end{tabular}

*Média \pm desvio-padrão; * *Mediana; IR:índice de resistência; IP: índice de pulsatilidade; PVS: pico de velocidade sistólica; RPV: razão entre picos de velocidade; VDF: velocidade diastólica final; DMG: diabetes mellitus gestacional.

A comparação entre as médias e medianas dos índices obtidos nos dois grupos está representada pelo nível de significância p.

gestacionais e pressão arterial, ambos com $\mathrm{p}=0,0$. Houve diferença entre as médias das idades maternas dos dois grupos, com $\mathrm{p}=0,7$. Entretanto, todas as gestantes eram jovens, com idade inferior a 36 anos, sem registro de doenças crônicas ou uso de medicações vasoativas.

Ao se comparar as médias e medianas dos índices doplervelocimétricos da artéria oftálmica entre os grupos de gestantes normais e com diabetes mellitus gestacional, não foram identificadas diferenças significantes (Tabela 1).

A mediana do pico de velocidade sistólica da artéria oftálmica foi de $33,6 \mathrm{~cm} / \mathrm{seg}$ no grupo de gestantes com DMG e no Grupo Controle foi de 31,9 cm/seg, sem diferenças significantes entre as medianas dos grupos, representado por $\mathrm{p}=0,7$. A velocidade diastólica final apresentou mediana de $6,3 \mathrm{~cm} / \mathrm{seg}$ e $7,9 \mathrm{~cm} / \mathrm{seg}$ nos grupos com DMG e Controle, respectivamente, sem diferenças significantes entre as medianas, com $\mathrm{p}=0,4$. As médias com desvio padrão da razão entre picos de velocidade foi de $0,5+/-0,1$ e $0,5+/ 0,1$ nos grupos com DMG e Controle, respectivamente, com $\mathrm{p}=0,4$, quando comparadas as médias.

Já as médias com desvio padrão dos índices de pulsatilidade dos Grupos Controle foram, respectivamente, $1,7 \pm 0,6$ e $1,6+/-0,4$, sem diferenças significantes entre os dois grupos, com $\mathrm{p}=0,7$. Finalmente as medianas dos índices de resistência foram semelhantes entre os dois grupos, com valor de 0,7 e p=0,9.

\section{Discussão}

A exposição das gestantes com DGM ao estado de hiperglicemia é limitada a um curto período. Já a hiperglicemia crônica do diabetes mellitus tipos 1 e 2 está associada a danos teciduais, disfunções e falhas em diferentes sistemas, especialmente nos olhos, rins, nervos, coração e vasos sanguíneos por meio de alterações funcionais e estruturais da micro e macrocirculação ${ }^{1}$.

Um dos componentes anatômicos das artérias que compõem a micro e macrovasculatura é o endotélio. Este é um tecido especializado relacionado a várias funções fisiológicas, incluindo a modulação do fluxo sanguíneo durante a homeostase e em condições de doenças ${ }^{9}$. A homeostase ocorre por meio da síntese de substâncias vasoativas que modulam o tônus vascular, inibem a agregação plaquetária e a proliferação das células musculares lisas vasculares ${ }^{10}$. Uma dessas substâncias importantes na homeostase vascular é o óxido nítrico9. Em situações de doenças, como diabetes mellitus, pode ocorrer o desacoplamento do óxido nítrico sintase endotelial, sendo esse um importante mecanismo inicial de disfunção endotelial ${ }^{9,11}$. Os principais determinantes da disfunção arterial são a diminuição da disponibilidade de ON e preponderância de fatores vasoconstritores liberados pelo endotélio, em detrimento aos fatores vasodilatadores, levando a um aumento do tônus vascular e vasoconstricção?.

A vasculopatia é a intercorrência frequente entre as pacientes diabéticas, e tem levado muitos pesquisadores a estudar a impedância vascular orbital por meio do emprego da doplervelocimetria das artérias retinianas ${ }^{12,13}$. O primeiro estudo da artéria oftálmica em gestantes foi publicado em $1992^{14}$. Os autores analisaram a impedância orbital por meio do registro do IP da artéria oftálmica de gestantes com hipertensão arterial transitória e hipertensão arterial crônica. Os autores encontraram valores mais baixos do IP nessas pacientes ao compará-las ao grupo normal, demonstrando sinais de hiperperfusão orbital, o que contribuiu para o melhor entendimento da adaptação vascular nesse território.

Como os índices Doppler da artéria oftálmica já estão bem estabelecidos no grupo de gestantes normais e não se modificam ao longo da gestação segundo a maioria dos autores, torna-se um método importante e já consagrado para o estudo do sistema nervoso central de gestantes ${ }^{15-17}$.

O interesse relativo à doplervelocimetria da artéria oftálmica em gestantes diabéticas deve-se à possibilidade de o método identificar alterações na microvascularização orbital, por meio da análise quantitativa da onda de velocidade de fluxo dessa artéria. Sabe-se que o estudo da hemodinâmica do sistema nervoso central em gestantes é tecnicamente limitado, por não ser permitido o uso de raio $\mathrm{X}$ ou métodos contrastados, colocando, assim, a doplervelocimetria como o de escolha para o estudo da hemodinâmica orbital e do sistema nervoso central, já que as artérias oftálmicas têm a mesma origem embrionária e mesmo comportamento funcional em relação às artérias do $\mathrm{SNC}^{18}$.

Como o diabetes mellitus gestacional expõe a paciente a riscos de desequilíbrio da função do endotélio e consequente alteração da homeostase arterial, foi motivo do nosso estudo tentar identificar se nesses casos é possível identificar modificações significativas do padrão vascular das artérias oftálmicas em gestantes atendidas no nosso ambulatório com DMG. Durante a revisão bibliográfica identificamos apenas um estudo, no qual foi avaliado o padrão Doppler da artéria oftálmica; porém, em grupo de 
gestantes com diabetes mellitus do tipo 1 comparando-as com gestantes normais. Foram detectados valores mais baixos de IP no grupo de gestantes com DM1 em relação a gestantes normais, evidenciando sinais de hiperfluxo nessas pacientes diabéticas ${ }^{8}$. Esses achados sugerem a existência de centralização do fluxo em gestantes DM1, provavelmente devido à distensão passiva das arteríolas dos órgãos em geral e edema vasogênico. Outra hipótese é que há instalação de disfunções endoteliais em gestantes DM1, com modificações da biodisponibilidade do óxido nítrico, causando vasodilatação cerebral materna, que precederiam a ocorrência de microangiopatia diabética.

Estudamos dois grupos de gestantes sem pareamento da idade. Entretanto, como se trata de análise do padrão arterial de gestantes adultas jovens, não é esperado que haja lesões vasculares crônicas que provoquem modificações importantes na reatividade vascular. Portanto, não acreditamos que isso possa ter influenciado na interpretação dos resultados.

Os resultados do atual estudo não demonstraram modificações significantes entre os índices doplervelocimétricos no grupo de gestantes com DMG em relação às gestantes normais. Esses achados podem sinalizar para o fato de que o DMG bem controlado não causa lesões endoteliais importantes e irreversíveis, como ocorre no DM1, bem como não é capaz de modificar a reatividade arterial no território central das gestantes.

Novos estudos sobre a reatividade da artéria oftálmica em gestantes diabéticas, por meio da técnica Doppler, serão necessários para definirmos se o tempo de doença e a descompensação glicêmica podem ser fatores associados à má resposta adaptativa arterial no território orbital.

\section{Referências}

1. American Diabetes Association. Diagnosis and classification of diabetes mellitus. Diabetes Care. 2012;35(Suppl 1):S64-71.

2. Schneider S, Bock C, Wetzel M, Maul H, Loerbroks A. The prevalence of gestational diabetes in advanced economies. J Perinat Med. 2012 Jun 12. [Ahead of print].

3. Dennedy MC, Avalos G, O'Reilly MW, O'Sullivan EP, Dunne FP. The impact of maternal obesity on gestational outcomes. Ir Med J. 2012;105(5 Suppl):23-5.

4. Ino-ue $M$, Azumi A, Yamamoto $M$. Ophthalmic artery blood flow velocity changes in diabetic patients as a manifestation of macroangiopathy. Acta Ophthalmol Scand. 2000;78(2):173-6.

5. Zhang H, Dellsperger KC, Zhang $C$. The link between metabolic abnormalities and endothelial dysfunction in type 2 diabetes: an update. Basic Res Cardiol. 2012;107(1):237-15.

6. Evensen AE. Update on gestational diabetes mellitus. Prem Care. 2012;39(1):83-94.

7. Diniz ALD, Moron AF, Santos MC, Sass N. Dopplervelocimetria colorida dos vasos orbitais: técnica de exame e anatomia vascular normal. Radiol Bras. 2004;37(4):287-90.

8. Hata T, YanagiharaT, Tanaka H, Yamashiro C, Kanenishi K. Maternal ophthalmic artery Doppler velocimetry in type 1 diabetes during pregnancy. Hum Reprod. 2000;15(1):222-3.

9. Palmer RM, Ferrige AG, Moncada S. Nitric oxide release accounts for the biological activity of endothelium-derived relaxing factor. Nature. 1987;327(6122):524-6.

10. Westermeier F, Puebla C, Vega JL, Farías M, Escudero C, Casanello $\mathrm{P}$, et al. Equilibrative nucleosid transporters in fetal endothelial dysfunction in diabetes mellitus and hyperglycaemia. Curr Vasc Pharmacol. 2009;7(4):435-49.

11. Nathan DM, Lachin J, Cleary P, Orchard T, Brillon DJ, Backlund JY, et al. Intensive diabetes therapy and carotid intima-media thickness in type 1 diabetes mellitus. N Engl J Med. 2003;348(23):2294-303.

12. Basturk T, Albayrak R, Ulas T, Akcay M, Unsal A, Toksoy M, et al. Evaluation of resistive index by color Doppler imaging of orbital arteries in type II diabetes mellitus patients with microalbuminuria. Ren Fail. 2012;34(6):708-12.

13. Lockhart CJ, McCann A, Agnew CA, Hamilton PK, Quinn CE, McClenaghan $\mathrm{V}$, et al. Impaired microvascular properties in uncomplicated type 1 diabetes identified by Doppler ultrasound of the ocular circulation. Diab Vasc Dis Res. $2011 ; 8(3): 211-20$.

14. Hata T, Senoh D, Hata K, Kitao M. Ophthalmic artery velocimetry in pregnant women. Lancet. 1992;340(8812): 182-3.

15. Diniz AL, Moron AF, Santos MC, Sass N, Pires CR, Debs CL. Ophthalmic artery Doppler as a measure of severe pre-eclampsia. Int J Gynaecol Obstet. 2008; 100(3):2 16-20.

16. Carneiro RS, Sass N, Diniz AL, Souza EV, Torloni MR, Moron AF. Ophthalmic artery Doppler velocimetry in healthy pregnancy. Int J Gynaecol Obstet. 2008; 100(3):21 1-5.

17. Diniz ALD, Moron AF, Santos MC, Sass N, Pires CR. Dopplervelocimetry of ophthalmic and central retinal arteries in normal pregnancies. Rev Bras Ginecol Obstet. 2005;27(4):168-73.

18. Belfort MA, Saade GR, Moise KJ Jr. The effect of magnesium sulfate on maternal retinal blood flow in preeclampsia: a randomized placebocontrolled study. Am J Obstet Gynecol. 1992;167(6):1548-53. 